\title{
Overexpression of REIC/Dkk-3 suppresses the expression of CD147 and inhibits the proliferation of human bladder cancer cells
}

\author{
YUHEI HORIKAWA $^{1}$, MASAMI WATANABE ${ }^{1,2}$, TAKUYA SADAHIRA ${ }^{1}$, \\ YUICHI ARIYOSHI $^{1}$, YASUYUKI KOBAYASHI ${ }^{1}$, MOTOO ARAKI ${ }^{1}$, KOICHIRO WADA $^{1}$, \\ KAZUHIKO OCHIAI ${ }^{3}$, SHUN-AI LI ${ }^{1,2}$ and YASUTOMO NASU ${ }^{1}$ \\ ${ }^{1}$ Department of Urology, Okayama University; ${ }^{2}$ Center for Innovative Clinical Medicine, \\ Okayama University Hospital, Okayama $700-8558 ;{ }^{3}$ Department of Veterinary Nursing and Technology, \\ Nippon Veterinary and Life Science University, Musashino, Tokyo 180-8602, Japan
}

Received December 5, 2016; Accepted April 25, 2017

DOI: $10.3892 / 01.2017 .6548$

\begin{abstract}
Our group previously developed an adenoviral vector encoding the REIC/Dkk-3 gene (Ad-REIC), a tumor suppressor, for cancer gene therapy. The Ad-REIC agent induces apoptosis and inhibits invasion in a number of cancer cell lines; however, the molecular mechanisms underlying its effects remain unclear. Cluster of differentiation 147 (CD147), also known as extracellular matrix metalloproteinase inducer (EMMPRIN), is a key molecule that promotes cancer proliferation and invasion. In order to elucidate the therapeutic mechanism of Ad-REIC, its effect on the expression of CD147 in human bladder cancer KK47 cells was investigated. Treatment with Ad-REIC markedly downregulated the expression of CD147 and significantly inhibited cellular proliferation. Since the expression of CD147 is reported to be under the positive control of mitogen-activated protein kinase (MAPK) signaling and the c-Myc protein, the correlations between the expression of CD147 and the activation of MAPKs or the expression of c-Myc were examined. Unexpectedly, no positive correlation was observed between the level of CD147 and the potential regulators that were assessed, indicating that another signaling pathway is responsible for the downregulation of CD147. The results from the present study demonstrate that Ad-REIC treatment can significantly downregulate the expression of CD147 in bladder cancer cells. Downregulation of the cancer-progression factor CD147 may be a novel mechanism that underlies the therapeutic effects of Ad-REIC treatment.
\end{abstract}

Correspondence to: Professor Masami Watanabe, Department of Urology, Okayama University, 2-5-1 Shikata-cho, Okayama 700-8558, Japan

E-mail: mwcorrespondence@gmail.com

Key words: bladder cancer, REIC/Dkk-3, CD147, EMMPRIN, cell proliferation

\section{Introduction}

Bladder cancer is one of the most common urothelial malignancies (1). Superficial bladder cancer can be treated by the transurethral resection of bladder tumors; however, 50-70\% of noninvasive cancers recur and $15-30 \%$ of cases progress to invasive muscle disease $(1,2)$. Although systemic chemotherapy is typically used in the treatment of the invasive bladder cancer, recurrence and progression rates remain high. Bladder cancer is therefore resistant to conventional therapeutic methods, and novel therapeutic agents for the treatment of bladder cancer are required (1).

The reduced expression in immortalized cells (REIC) gene is also known as Dickkopf-3 (Dkk-3). A previous study from our group reported that REIC/Dkk-3 is a tumor suppressor gene that may be a target for future treatments (2). The expression of REIC/Dkk-3 is downregulated in a wide range of human cancer types, including bladder cancer, which suggests that REIC/Dkk-3 is a promising therapeutic gene $(2,3)$. With the expectation that the overexpression of REIC/Dkk-3 could promote anticancer effects, our group previously developed an adenovirus vector encoding the human REIC/Dkk3 gene (Ad-REIC) and demonstrated that the Ad-REIC agent induced cancer-specific apoptosis in a number of cancer cell lines (4), and the phosphorylation of c-Jun N-terminal kinase (JNK) was revealed to be an important step in inducing cancer cell apoptosis (3-5). In addition, Ad-REIC inhibits cell motility and invasion in various cancer cells; however, the molecular mechanisms underlying these multiple therapeutic effects remain unclear (4).

Cluster of differentiation (CD)147 [also known as extracellular matrix metalloproteinase inducer (EMMPRIN) or basigin] is a cell surface glycoprotein belonging to the immunoglobulin superfamily. CD147 was demonstrated to be the most frequently upregulated mRNA and protein in micro-metastatic cells isolated from the bone marrow of patients with cancer, suggesting that it serves a key role in tumorigenesis and metastasis (6). The expression of the CD147 protein in cancer cells, including urinary bladder, breast, lung, oral cavity, esophageal 
and skin cancer, as well as malignant lymphoma cells, has been observed to be significantly increased compared with that in the corresponding normal tissue (6). The elevated expression of CD147 is correlated with tumor progression in bladder cancer, glioma, giant cell tumor of bone, laryngeal squamous cell carcinoma, ovarian carcinoma and melanoma, indicating that CD147 serves a significant role in the progression of cancer (6-11). It has previously been demonstrated that CD147 is involved in the cell glycolytic metabolic pathways that enable cancer cells to divide and rapidly proliferate (12-14), providing an insight into the molecular mechanisms underlying the role that CD147 serves in cancer progression. In addition, the high expression of CD147 on the surface of cancer cells stimulates adjacent cancer cells and fibroblasts to produce matrix metalloproteinases (6).

Since CD147 has rapidly emerged as a strong promoter of the development and progression of cancer, the present study investigated the regulation of CD147 expression by the Ad-REIC-mediated therapeutic overexpression of REIC/Dkk-3, and examined the signaling pathways associated with the downregulation of CD147.

\section{Materials and methods}

Cells and cell culture. Human urothelial cancer cell lines (HT1376, RT4, T24, UMUC3, J82, 5637, TCCsup), a mouse bladder cancer cell line (MBT2) and human embryonic kidney cells (HEK293) were obtained from the American Type Culture Collection (Manassas, VA, USA). The KK47 human bladder cancer cell line was kindly provided by Professor S. Naito (Department of Urology, University of Kyushu, Fukuoka, Japan) (15). The cells were grown in RPMI-1640 medium (Sigma-Aldrich; Merck KGaA, Darmstadt, Germany) supplemented with $10 \%$ (v/v) fetal bovine serum (FBS) (Invitrogen; Thermo Fisher Scientific, Inc., Waltham, MA, USA), penicillin (100 IU/ml) and streptomycin $(100 \mu \mathrm{g} / \mathrm{ml})(2)$. Human normal bladder urothelial cells (HUC; cat. no. 4320) were purchased from ScienCell ${ }^{\mathrm{TM}}$ Research Laboratories, Inc. (San Diego, CA, USA) and were cultivated using the medium recommended by the supplier (16).

Construction and production of Ad-REIC. In order to generate Ad-REIC vectors, pShuttle-SGE-REIC plasmids were digested with the restriction enzymes I-CeuI and PI-SceI and inserted into the Adeno-X Viral DNA (Clontech Laboratories, Inc., Mountainview, CA, USA), as previously described $(3,17)$. Briefly, the recombinant adenoviral DNA with the full-length human REIC/Dkk-3 gene was linearized by digestion with PacI and transfected into HEK293 cells. At 7-10 days following transfection, the HEK293 cells were harvested, and a viral solution was obtained by three freeze/thaw cycles. The recovered virus solution was used to propagate sufficient viruses in HEK293 cells for further studies. All virus particles were purified by $\mathrm{CsCl}$ density gradient ultracentrifugation and were stored at $-80^{\circ} \mathrm{C}$. An adenovirus vector carrying the LacZ gene (Ad-LacZ) was used as a control, as described previously $(3,5)$. The adenoviral vectors were produced using replication-defective adenoviruses of serotype 5 .

Assay for human telomerase reverse transcriptase (hTERT) promoter-driving activity and CD147 band density. The hTERT promoter-driving activity was analyzed as previously described (16). HUC, HT1376, RT4, T24, UMUC3, J82, 5637, KK47, TCCsup and MBT2 cells $\left(4 \times 10^{5}\right)$ were plated in 6-well plates in culture medium containing charcoal-stripped FBS. The cells were cultured for $24 \mathrm{~h}$, and the transient transfection of the luciferase-encoding plasmid with the advanced two-step transcriptional amplification (TSTA) system were performed using the Lipofectamine transfection reagent (Invitrogen; Thermo Fisher Scientific, Inc.). The advanced TSTA system is a transcriptional system that can robustly enhance the hTERT promoter-driven and cancer-specific expression of genes (16). In the luciferase assay, the effector plasmid was co-transfected with the reporter plasmid, which was derived from the Dual-Luciferase Reporter Assay kit (Promega Corporation, Madison, WI, USA). The cells were incubated for $48 \mathrm{~h}$, harvested and assayed for luciferase activity using the luciferase assay kit and a luminescence microplate reader, according to the manufacturer's protocol.

Western blotting. Western blotting was performed as previously described $(2,3,17)$. KK47 cells $\left(5 \times 10^{5}\right)$ were seeded in flat-bottom 6-well plates, incubated for $24 \mathrm{~h}$ and then sampled. In some experiments, the cells were treated with Ad-LacZ or Ad-REIC at a multiplicity of infection (MOI) of 100 in $0.5 \mathrm{ml}$ of complete medium for $1 \mathrm{~h}$, prior to the addition of $1.5 \mathrm{ml}$ of fresh medium and incubation of the cells for $24 \mathrm{~h}$. Subsequently, the floating dead cells were removed and attached cells were lysed with ice-cold lysis buffer to extract proteins. The insoluble fragments were removed by centrifugation and the supernatants were adjusted to an equal protein concentration in each experiment. The samples were separated on a 7.5\% SDS-PAGE gel and transferred onto a polyvinylidene fluoride membrane (EMD Millipore, Billerica, MA, USA) for western blotting. Following the transfer, the membranes were blocked for $1 \mathrm{~h}$ with $5 \%$ nonfat milk powder, $6 \%$ glycine and $0.1 \%$ Tween-20 in Tris-buffered saline (TBST) at room temperature. The membranes were incubated for $1 \mathrm{~h}$ at room temperature with the following primary antibodies at 1:1,000 dilution: CD147 (cat. no., EPR4052; Abcam, Cambridge, UK), REIC/Dkk-3 (mouse monoclonal, raised in our laboratory) (17), phospho-p38 mitogen-activated protein kinase (MAPK; cat. no. 4511), phospho-p44/42 MAPK (Erk1/2; cat. no. 4370), phospho-JNK (cat. no. 9251), phospho-c-Jun (cat. no. 2361), c-Myc (cat. no. 9402) and $\beta$-actin (cat. no. 4967; all from Cell Signaling Technology, Inc., Danvers, MA, USA). After three washes in TBST, the membranes were incubated with a horseradish peroxidase-conjugated secondary antibody (dilution, 1:5,000; cat. no., NA931; GE Healthcare Life Sciences, Little Chalfont, UK) for $1 \mathrm{~h}$ at room temperature. The bound antibodies were visualized by the enhanced chemiluminescence detection method (Amersham ECL kit; GE Healthcare Life Sciences) using medical X-ray film (2). In order to determine band density by western blotting, the films were scanned and a densitometric analysis was performed using ImageJ software (version 1.44p, available from: https://imagej.nih.gov/ij/; National Institutes of Health, Bethesda, MD, USA).

Cell viability assay. KK47 bladder cancer cells $\left(5 \times 10^{5}\right)$ were seeded in 6-well plates and incubated for $24 \mathrm{~h}$ at $37^{\circ} \mathrm{C}$. The cells were treated with Ad-LacZ or Ad-REIC at $100 \mathrm{MOI}$ in $0.5 \mathrm{ml}$ 
of complete medium for $1 \mathrm{~h}, 1.5 \mathrm{ml}$ of fresh medium was added, and the cells were then incubated for $72 \mathrm{~h}$. Following removal of the floating dead cells by exchanging medium, the attached cells were detached by trypsin and the number of cells was counted using a disposable hemocytometer without staining.

Statistical analysis. The data are presented as the mean \pm standard deviation from five experiments. The unpaired Student's t-test was performed to analyze the statistical significance of differences between two groups. A regression analysis was performed to examine the correlation between two parameters. $\mathrm{P}<0.05$ was considered to indicate a statistically significant difference. Statistical analyses were performed using StatView version 4.5 software (Abacus Concepts, Piscataway, NJ, USA).

\section{Results}

Expression of CD147 in human urothelial cancer cell lines. The expression of CD147 protein was first examined by western blotting in a number of urothelial cancer cell lines and a human normal bladder urothelial cell line (HUC). In the majority of the urothelial cancer cell lines investigated, the $\mathrm{CD} 147$ protein bands were identified at a molecular weight of 50-60 kDa (Fig. 1A); as previously reported, the protein exhibited multiple bands of different molecular sizes (6). In the KK47 human bladder cancer cell line, the expression level of CD147 was markedly increased compared with that in other urothelial cancer cell lines (Fig. 1A). CD147 expression was not observed in the HUC cells.

Correlation between the expression of CD147 and the hTERT promoter-driving activity in human urothelial cells. Since CD147 functions as an oncoprotein (6-11), the association between CD147 expression and malignancy-associated factors was examined in a number of urothelial cancer cell lines and normal HUC cells. The hTERT promoter-driving activity was measured as an index of the malignancy using the hTERT promoter-driven advanced TSTA system (16). A significant correlation was observed between the CD147 band density and the value of the hTERT promoter-driving activity, as determined by the regression analysis (Fig. 1B). The results indicated that high CD147 expression levels were positively associated with the promoter-driving activity of hTERT, and were correlated with hTERT expression levels and cancer malignancy (16).

Ad-REIC treatment downregulates the expression of CDI47 in human bladder cancer KK47 cells. The expression levels of REIC/Dkk-3 and CD147 following Ad-REIC treatment were examined by western blotting (Fig. 2). REIC/Dkk-3 protein was expressed following Ad-REIC but not Ad-LacZ treatment. The expression of CD147 was clearly observed in untreated and Ad-LacZ-treated KK47 cells, whereas Ad-REIC treatment markedly downregulated the expression of CD147 in these cells.

Ad-REIC significantly reduces the viability of KK47 bladder cancer cells. KK47 cancer cells were treated with Ad-REIC to assess the anti-proliferative effects of the agent. A marked
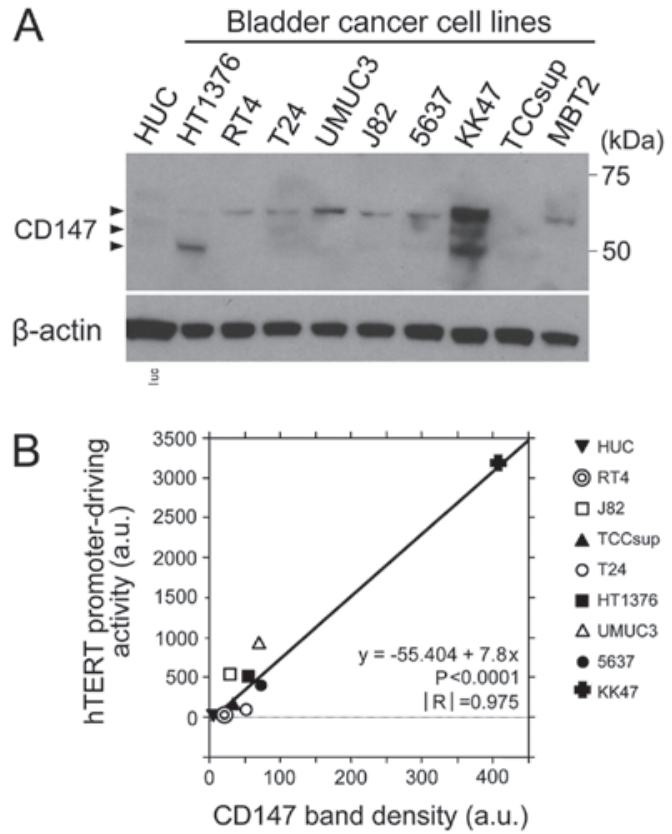

Figure 1. Expression of CD147 in human urothelial cancer cell lines. (A) Western blot analysis of CD147 expression in human and mouse urothelial cell lines. Human normal bladder urothelial cells (HUC) and various bladder cancer cell lines were examined. $\beta$-actin was used as a loading control (B) Correlation analysis between the expression of CD147 and the hTERT promoter-driving activity in human urothelial cells. The band density in was determined by a densitometric analysis. The hTERT promoter-driving activity was measured as an index of malignancy using an hTERT promoter-dependent expression system. CD147, cluster of differentiation 147; hTERT, human telomerase reverse transcriptase; a.u., arbitrary unit.

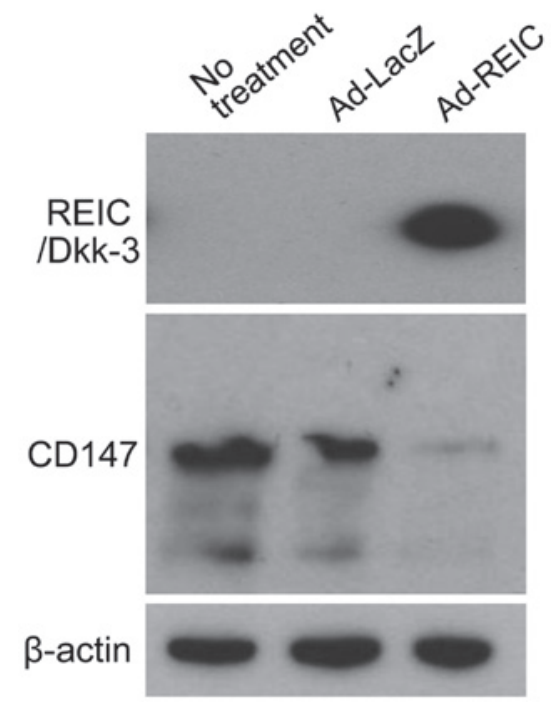

Figure 2. Ad-REIC treatment downregulates the expression of CD147 in human bladder cancer KK47 cells. The overexpression of REIC/Dkk-3 was induced by the Ad-REIC agent at $100 \mathrm{MOI}$ and the expression level of the indicated proteins was analyzed by western blotting. $\beta$-actin was used as a loading control. REIC/Dkk3, reduced expression in immortalized cells/Dickkopf-3; CD147, cluster of differentiation 147; Ad-REIC, adenoviral vector encoding the human REIC/Dkk3 gene.

induction of apoptosis was observed in the Ad-REIC treatment group in comparison to the other treatment groups (Fig. 3A). The attached viable cells were counted following removal of floating dead cells. There number of cells in the Ad-REIC 

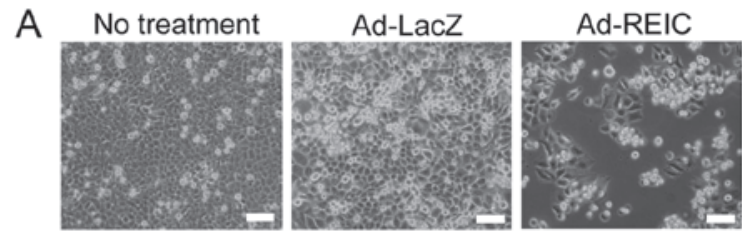

Bar $=100 \mu \mathrm{m}$

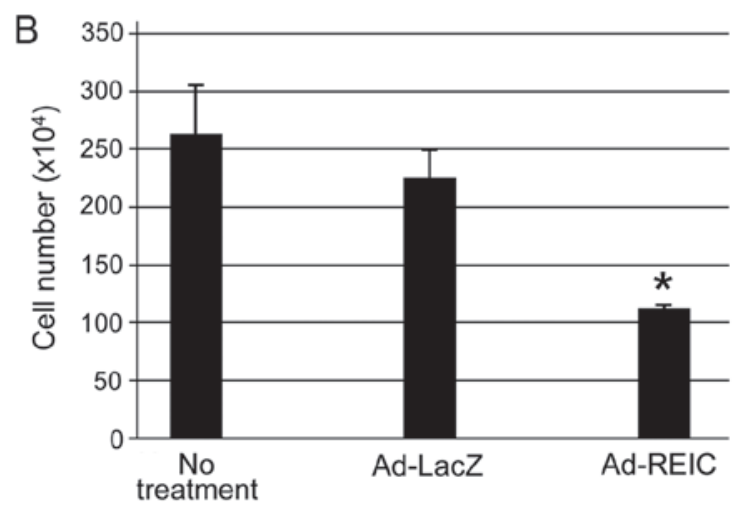

Figure 3. Inhibition of cell proliferation in KK47 human bladder cancer cells following Ad-REIC treatment. (A) The appearance of the cells after the indicated treatment is shown by phase contrast microscopy. (B) Bar graph of the number of cells following the treatment. Five experiments were performed A significant difference was observed ( $\mathrm{P}<0.05)$ between the Ad-REIC and the control Ad-LacZ treatments. REIC/Dkk3, reduced expression in immortalized cells/Dickkopf-3; Ad-REIC, adenoviral vector encoding the human REIC/Dkk3 gene.

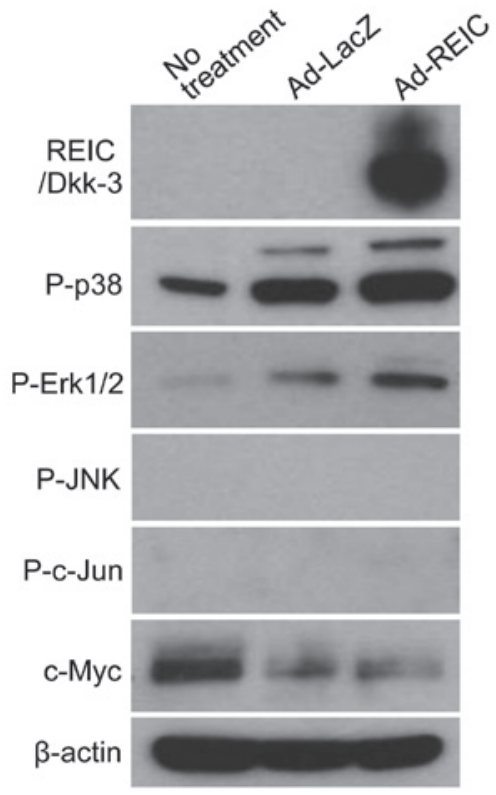

Figure 4. Expression levels of the possible factors regulating the expression of CD147 in KK47 cells following Ad-REIC treatment. Since the expression of CD147 is reported to be under the positive control of p38-, Erk1/2- and JNK-dependent MAPK signaling and the expression of c-Myc, these proteins were analyzed by western blotting. $\beta$-actin was used as a loading control CD147, cluster of differentiation 147; REIC/Dkk3, reduced expression in immortalized cells/Dickkopf-3; Ad-REIC, adenoviral vector encoding the human REIC/Dkk3 gene; p38, p38 MAPK; Erk1/2, extracellular signal-regulated kinase 1/2; JNK, c-Jun N-terminal kinase; MAPK, mitogen-activated protein kinase.

treatment group was significantly lower compared with that in than the other groups $(\mathrm{P}<0.05$; Fig. $3 \mathrm{~B})$, indicating that
Ad-REIC significantly decreased cell viability in the bladder cancer cell line.

Ad-REIC downregulates the expression of CD147 without dependence on the MAPK and c-Myc signaling pathway. With regard to the regulation of the expression of CD147, several signaling pathways are reported to be associated with its gene transcription (18-23). In particular, the expression of CD147 is reported to be positively regulated by p38-, Erk1/2and JNK-dependent MAPK signaling pathways and the c-Myc protein $(18,19,21,22)$. The associations between CD147 expression, phosphorylation of MAPK pathway components, and the expression of c-Myc following Ad-REIC treatment was examined. In addition, since JNK-dependent signaling has been reported to be a critical step in Ad-REIC-induced cancer cell death $(4,5)$, the phosphorylation of JNK and c-Jun was investigated. Western blotting was performed to analyze the expression levels of the indicated factors following Ad-LacZ and Ad-REIC treatment (Fig. 4). The expression of REIC/Dkk-3 protein was confirmed following Ad-REIC treatment. Unexpectedly, no positive association was observed between CD147 level and MAPK signaling or c-Myc expression. MAPK signaling via p38 and Erk $1 / 2$ was markedly activated following Ad-REIC treatment, indicating that the agent affected the intrinsic cell survival response against cell death signaling. The results indicated that the downregulation of CD147 by Ad-REIC occurs without the inhibition of MAPK signaling or the suppression of c-Myc. Furthermore, the levels of phosphorylated JNK and c-Jun were not upregulated following Ad-REIC treatment, indicating that JNK signaling was not significantly activated by this agent in KK47 bladder cancer cells.

\section{Discussion}

The expression of CD147 is significantly upregulated in a number of types of malignancy (6). It is a key molecule that promotes the proliferation, invasion and metastasis of cancer cells (6-11). CD147 is an important promoter of cell growth through the reprogramming of glucose metabolism, including glycolysis (12,24-26). These findings indicate that cancer cells use the CD147-dependent glycolytic metabolic pathways in order to divide and rapidly proliferate (12-14). Thus, inhibiting or blocking the expression of CD147 to reduce the glycolytic capacity of the targeted cancer cells represents an attractive cancer therapeutic strategy (27-29). In order to further elucidate the therapeutic mechanism of Ad-REIC agents for cancer gene therapy, the effects of Ad-REIC on the expression of CD147 and its associated signaling molecules in human bladder cancer cells were investigated in the present study. In vitro Ad-REIC treatment was revealed to significantly downregulate the expression of CD147 and to inhibit the proliferation of bladder cancer cells, which suggests an association between the downregulation of CD147 and the anti-cancer effects of the agents.

The molecular mechanisms underlying Ad-REIC-induced apoptosis have been previously investigated and the phosphorylation (activation) of JNK was demonstrated to be a critical step in cancer cell death (5). REIC/Dkk-3 protein is a secretory protein and its overexpression in response to Ad-REIC treatment efficiently leads to endoplasmic reticulum (ER) 
stress-induced apoptosis in cancer cells (4). ER stress-induced apoptosis is triggered due to a failure in the folding of large amounts of REIC/Dkk-3 protein in the lumen of the ER, and the phosphorylation of JNK occurs downstream of ER stress signaling $(4,5)$. The levels of phosphorylated JNK and c-Jun following Ad-REIC treatment in bladder cancer cells were therefore analyzed in the present study. Surprisingly, treatment with the Ad-REIC agent did not lead to activation of JNK signaling in KK47 cells. The induction of apoptosis and the inhibition of proliferation were observed in KK47 cells, indicating that the activation of JNK may not be essential for the anti-proliferative effects of Ad-REIC. It is possible that cancer suppressive mechanisms other than JNK signaling may underlie the effects of Ad-REIC and that CD147 may be a novel target of cancer therapeutic agents. Different cancer cells have their own genetic characterization; however, the same phenomenon has been observed in human prostate cancer LNCap cell lines (17).

With regard to the regulation of CD147 expression, several signaling pathways are reported to be associated with its transcription (18-23). The core promoter and transcription factor-binding sites of CD147 were previously analyzed and identified as targets of the cancer-associated transcription factors c-Myc and Sp1 $(22,23)$. Since the expression of CD147 is known to be positively regulated by p38-, Erk1/2- and JNK-dependent MAPK signaling and c-Myc protein, the associations between CD147 expression, MAPK pathway activation and the expression of c-Myc following Ad-REIC treatment were examined in the present study. Unexpectedly, no positive correlation was observed between the expression of CD147 and the possible regulators that were assessed. These results indicate that another signaling pathway that was not examined in the present study could be responsible for the downregulation of CD147.

Our group recently conducted a phase I/IIa study of in situ gene therapy for patients with prostate cancer, using an Ad-REIC agent produced according to good manufacturing practice guidelines (30). Patients with hormonal therapy-resistant prostate cancer with or without metastasis were enrolled and evaluated to investigate the tumor inhibitory effects of Ad-REIC treatment. The survival time of one patient with progressive lymph node metastases was favorable, and significant tumor killing effects were demonstrated in Ad-REIC-injected lesions, with apparent reductions in tumor volume (31). Although experimental studies with cancer-bearing mouse models are required to investigate the downregulation of CD147 in Ad-REIC-injected tumors, the therapeutic effects observed in the treated legions could be partially explained by the inhibition of the CD147 oncoprotein by the Ad-REIC agent.

In conclusion, a novel therapeutic mechanism underlies the effects of the Ad-REIC agent developed for cancer gene therapy. In addition to the previously reported activation of the JNK signaling pathway (5), the downregulation of the cancer-progression factor CD147 could be one of the major therapeutic effects of Ad-REIC gene therapy. The mechanisms and the variety of roles of CD147 in cancer progression make the oncoprotein an attractive target in the field of cancer therapy. Further studies are required to elucidate the mechanisms that regulate the expression of CD147.

\section{Acknowledgements}

This study was supported by scientific research grants [Japan Society for the Promotion of Science Grants-in-Aid for Scientific Research (KAKENHI); grant nos. JP15H04297, JP15K10590 and JP16K11004] from the Ministry of Education, Culture, Sports, Science and Technology of Japan and Takeda Science Foundation. The authors would like to thank Mr. Hideo Ueki and Ms. Fusaka Oonari (Okayama University) for their valuable assistance. Okayama University and Momotaro-Gene Inc. are applying for patents on the Ad-REIC agents. Drs Masami Watanabe and Yasutomo Nasu are the inventors of the patents and own stock in Momotaro-Gene Inc.

\section{References}

1. Dobruch J and Herr H: Should all patients receive single chemotherapeutic agent instillation after bladder tumour resection? BJU Int 104: 170-174, 2009.

2. Hirata T, Watanabe M, Kaku H, Kobayashi Y, Yamada H, Sakaguchi M, Takei K, Huh NH, Nasu Y and Kumon H: REIC/Dkk-3-encoding adenoviral vector as a potentially effective therapeutic agent for bladder cancer. Int J Oncol 41: 559-564, 2012.

3. Watanabe M, Sakaguchi M, Kinoshita R, Kaku H, Ariyoshi Y, Ueki H, Tanimoto R, Ebara S, Ochiai K, Futami J, et al: A novel gene expression system strongly enhances the anticancer effects of a REIC/Dkk-3-encoding adenoviral vector. Oncol Rep 31: 1089-1095, 2014.

4. Watanabe M, Nasu Y and Kumon H: Adenovirus-mediated REIC/Dkk-3 gene therapy: Development of an autologous cancer vaccination therapy (Review). Oncol Lett 7: 595-601, 2014.

5. Abarzua F, Sakaguchi M, Takaishi M, Nasu Y, Kurose K, Ebara S, Miyazaki M, Namba M, Kumon H and Huh NH: Adenovirus-mediated overexpression of REIC/Dkk-3 selectively induces apoptosis in human prostate cancer cells through activation of c-Jun-NH2-kinase. Cancer Res 65: 9617-9622, 2005.

6. Nabeshima K, Iwasaki H, Koga K, Hojo H, Suzumiya J and Kikuchi M: Emmprin (basigin/CD147): Matrix metalloproteinase modulator and multifunctional cell recognition molecule that plays a critical role in cancer progression. Pathol Int 56: 359-367, 2006.

7. Zhong WD, Chen QB, Ye YK, Han ZD, Bi XC, Dai QS Liang YX, Zeng GH, Wang YS, Zhu G, et al: Extracellular matrix metalloproteinase inducer expression has an impact on survival in human bladder cancer. Cancer Epidemiol 34: 478-482, 2010.

8. Pan Y, He B, Chen J, Sun H, Deng Q, Wang F, Ying H, Liu X, Lin K, Peng H, et al: Gene therapy for colorectal cancer by adenovirus-mediated siRNA targeting CD147 based on loss of the IGF2 imprinting system. Int J Oncol 47: 1881-1889, 2015.

9. Bovenzi CD, Hamilton J, Tassone P, Johnson J, Cognetti DM, Luginbuhl A, Keane WM, Zhan T, Tuluc M, Bar-Ad V, et al: Prognostic indications of elevated MCT4 and CD147 across cancer types: A meta-analysis. Biomed Res Int 2015: 242437, 2015.

10. Hatanaka M, Higashi Y, Kawai K, Su J, Zeng W, Chen X and Kanekura T: CD147-targeted siRNA in A375 malignant melanoma cells induces the phosphorylation of EGFR and downregulates cdc25C and $\mathrm{MEK}$ phosphorylation. Oncol Lett 11: 2424-2428, 2016.

11. Liang YX, Lu JM, Mo RJ, He HC, Xie J, Jiang FN, Lin ZY, Chen YR, Wu YD, Luo HW, et al: E2F1 promotes tumor cell invasion and migration through regulating CD147 in prostate cancer. Int J Oncol 48: 1650-1658, 2016.

12. Le Floch R, Chiche J, Marchiq I, Naiken T, Ilc K, Murray CM, Critchlow SE, Roux D, Simon MP and Pouysségur J: CD147 subunit of lactate/H+ symporters MCT1 and hypoxia-inducible MCT4 is critical for energetics and growth of glycolytic tumors. Proc Natl Acad Sci USA 108: 16663-16668, 2011.

13. Aït-Ali N, Fridlich R, Millet-Puel G, Clérin E, Delalande F, Jaillard C, Blond F, Perrocheau L, Reichman S, Byrne LC, et al: Rod-derived cone viability factor promotes cone survival by stimulating aerobic glycolysis. Cell 161: 817-832, 2015.

14. Marchiq I, Albrengues J, Granja S, Gaggioli C, Pouysségur J and Simon MP: Knock out of the BASIGIN/CD147 chaperone of lactate/H+ symporters disproves its pro-tumour action via extracellular matrix metalloproteases (MMPs) induction. Oncotarget 6: 24636-24648, 2015. 
15. Kimiya K, Naito S, Soejima T, Sakamoto N, Kotoh S, Kumazawa $\mathbf{J}$ and Tsuruo T: Establishment and characterization of doxorubicin-resistant human bladder cancer cell line, KK47/ADM. J Urol 148: 441-445, 1992.

16. Ueki H, Watanabe M, Kaku H, Huang P, Li SA, Ochiai K, Hirata T, Noguchi H, Yamada H, Takei K, et al: A novel gene expression system for detecting viable bladder cancer cells. Int $\mathrm{J}$ Oncol 41: 135-140, 2012.

17. Mori A, Watanabe M, Sadahira T, Kobayashi Y, Ariyoshi Y, Ueki H, Wada K, Ochiai K, Li SA and Nasu Y: The downregulation of the expression of $\mathrm{CD} 147$ by tumor suppressor REIC/Dkk-3, and its implication in human prostate cancer cell growth inhibition. Acta Med Okayama 71: 135-142, 2017.

18. Hagemann T, Wilson J, Kulbe H, Li NF, Leinster DA, Charles K, Klemm F, Pukrop T, Binder C and Balkwill FR: Macrophages induce invasiveness of epithelial cancer cells via NF-kappa B and JNK. J Immunol 175: 1197-1205, 2005.

19. Huang Z, Wang C, Wei L, Wang J, Fan Y, Wang L, Wang Y and Chen T: Resveratrol inhibits EMMPRIN expression via P38 and ERK1/2 pathways in PMA-induced THP-1 cells. Biochem Biophys Res Commun 374: 517-521, 2008.

20. Ge H, Zhang JF, Guo BS, He Q, Wang BY, He B and Wang CQ Resveratrol inhibits macrophage expression of EMMPRIN by activating PPARgamma. Vascul Pharmacol 46: 114-121, 2007.

21. Liu H, Xu XF, Zhao Y, Tang MC, Zhou YQ and Gao FH: NS-398 promotes pancreatic cancer cell invasion by CD147 and MMP-2 via the activation of P38. Mol Med Rep 13: 2208-2214, 2016.

22. Kong LM, Liao CG, Zhang Y, Xu J, Li Y, Huang W, Zhang Y, Bian $\mathrm{H}$ and Chen $\mathrm{ZN}$ : A regulatory loop involving miR-22, Sp1, and c-Myc modulates CD147 expression in breast cancer invasion and metastasis. Cancer Res 74: 3764-3778, 2014.

23. Liang L, Major T and Bocan T: Characterization of the promoter of human extracellular matrix metalloproteinase inducer (EMMPRIN). Gene 282: 75-86, 2002.
24. Baba M, Inoue M, Itoh K and Nishizawa Y: Blocking CD147 induces cell death in cancer cells through impairment of glycolytic energy metabolism. Biochem Biophys Res Commun 374: 111-116, 2008.

25. Huang Q, Li J, Xing J, Li W, Li H, Ke X, Zhang J, Ren T, Shang Y, Yang H, et al: CD147 promotes reprogramming of glucose metabolism and cell proliferation in HCC cells by inhibiting the p53-dependent signaling pathway. J Hepatol 61: 859-866, 2014

26. Marchiq I, Le Floch R, Roux D, Simon MP and Pouyssegur J: Genetic disruption of lactate/H+ symporters (MCTs) and their subunit CD147/BASIGIN sensitizes glycolytic tumor cells to phenformin. Cancer Res 75: 171-180, 2015.

27. Muramatsu T: Basigin (CD147), a multifunctional transmembrane glycoprotein with various binding partners. J Biochem 159: 481-490, 2016.

28. Fu ZG, Wang L, Cui HY, Peng JL, Wang SJ, Geng JJ, Liu JD, Feng F, Song F, Li L, et al: A novel small-molecule compound targeting CD147 inhibits the motility and invasion of hepatocellular carcinoma cells. Oncotarget 7: 9429-9447, 2016.

29. Walter M, Simanovich E, Brod V, Lahat N, Bitterman H and Rahat MA: An epitope-specific novel anti-EMMPRIN polyclonal antibody inhibits tumor progression. Oncoimmunology 5: e1078056, 2015.

30. Kumon H, Ariyoshi Y, Sasaki K, Sadahira T, Araki M, Ebara S, Yanai $\mathrm{H}$, Watanabe $\mathrm{M}$ and Nasu Y: Adenovirus vector carrying REIC/Dkk-3 gene: Neoadjuvant intraprostatic injection for high-risk localized prostate cancer undergoing radical prostatectomy. Cancer Gene Ther 23: 400-409, 2016.

31. Kumon H, Sasaki K, Ariyoshi Y, Sadahira T, Ebara S, Hiraki T, Kanazawa S, Yanai H, Watanabe M and Nasu Y: Ad-REIC gene therapy: Promising results in a patient with metastatic CRPC following chemotherapy. Clin Med Insights Oncol 9: 31-38, 2015. 